\begin{tabular}{lc}
\hline SCIENCE \& TECHNOLOGY \\
Journal homepage: http://www.pertanika.upm.edu.my/ \\
\hline PERTANIKA
\end{tabular}

\title{
Participatory Design: Apps from The Older Adults to The Older Adults
}

\section{Zaidatol Haslinda Abdullah Sani*, Dinna@Ninna Mohd Nizam and Aslina Baharum}

Faculty of Computing and Informatics, Universiti Malaysia Sabah, Labuan International Campus, 87000 Labuan FT, Malaysia

\begin{abstract}
The use of technology to address health issues among older adults is becoming popular nowadays, but, in practice, there is very little systematic work on how to design and develop for older adults. This paper investigated participatory design in designing and developing two mobile apps to support community-living older adults to maintain their health. We examine 1) three older adults to individually participate in designing an app to self-monitor their fruit, vegetable and liquid intakes and 2) a group of four older adults participate in designing an app to address loneliness. In this paper, we present methodological insights of conducting participatory design with older adults. We focus more on the mutual learning between the researcher and the older adults as "designers". We found that both methods provide rich data for developing the apps. However, when having a group of older adults together was found to stimulate the discussion among them easily, the participants were more open to critique the design suggestions, the moderator did not have to provoke often to guide the discussion, and in terms of time, although the session was slightly longer, it generates more data per participant. We acknowledge that the topic between the two groups was different, and each topic's privacy was also a concern. We also acknowledge that the number of participants is low, and the

ARTICLE INFO

Article history:

Received: 13 May 2021

Accepted: 19 August 2021

Published: 22 October 2021 participant's technology background can be a concern. Either way, we recommend continuing to involve older adults in the technology design and development phase.
\end{abstract}

DOI: https://doi.org/10.47836/pjst.29.4.35

E-mail addresses:

linda.sani@ums.edu.my (Zaidatol Haslinda Abdullah Sani) dinna@ums.edu.my (Dinna@Ninna Mohd Nizam)

aslina@ums.edu.my (Aslina Baharum)

* Corresponding author
Keywords: Evaluation, participatory design, user experience 


\section{INTRODUCTION}

Defining when one becomes old is difficult. The chronological age of 65 years or over is often the definition of older adults, as this has become the retirement age in most countries. However, not all countries have the same retirement age. Develop countries, for example, the United States, currently retires at age 66. In developing countries, such as Malaysia, the retirement age is slightly lower, which is either $55,56,58$, or 60 , depends solely on the employee themselves when they want to retire. Moreover, in some countries, the retirement age differs between genders. For example, in China, the men retire at age 60 years, and the women retire at age 50 to 55 years. Thus, with respect to all nations, the World Health Organization (WHO) and the United Nations (UN) use 60 years and above to define older adults in their reports. The population of older adults aged 60 years and above worldwide is multiplying (WHO, 2021). It is predicted that by 2050, the older adult population will double the current amount (WHO, 2021).

In Malaysia, however, the Department of Statistics Malaysia (DOSM) refers to an older adult as one age 65 years and above. Older adults increased from 1.75 million (5\%) in 2010 to 2.3 million (7\%) in 2020. The number is estimated to increase to 3.49 million (14.5\%) in 2040 (DOSM, 2020). The DOSM has also projected that in 2040 the younger population age below 14 years old to decrease from $27.4 \%$ in 2010 to $18.6 \%$ in 2040 . DOSM also predicted that the old-age support ratio, the number of working adults aged 15 to 64 years to older adults, will triple from 7.4 in 2010 to 21.7 in 2040 (DOSM, 2020). It shows a growing need to support older adults to remain independent in their later life.

The rising of the older adult population has raised concerns regarding their health conditions as their health tends to decline easily. Common health issues among older adults are malnutrition, dehydration and loneliness (BDA, 2021). It is known that the solution to treat any health issues is through healthy eating with well-balanced nutrition. In regard to loneliness, bereavement or children moving away from the family home are the most common reasons why older adults feel lonely in their later age. Moreover, older adults would not want to have health issues as these feelings are private and personal (Sani et. Al., 2020).

The rising of the older adult population and the low number of younger adults raised concerns that we need to ensure that the older adults will have enough support to live on their own in their later life. In regard to this, technology has become a promising tool to promote healthy living among older adults. There is a growth of gerontology studies to assist older adults to promote healthy attitudes and behaviour among older adults. Several technologies have been developed to assist older adult's health issues apart from taking medicines. For example, Tulu et al. (2016) developed an app for diabetic older adults to self-manage their daily life. Hakobyan et al. (2016) designed and developed a diet diary app for older adults with age-related macular degeneration. Sani and Petrie (2017) designed 
and developed an app for the community living older adults to self-monitor their food and liquid intakes. Mehra et al. (2018) developed an app to guide and monitor older adults exercising from afar. All of these examples have the same aim, which is to assist older adults to self-monitor themselves.

\section{Participatory Design}

Designing an app for older adults is not an easy task. This population group needs specific skills and guidelines prior to designing such technology for them. Participatory design is one method that engages all users of such technology to raise concerns, provide ideas and suggestions in designing such technology (DiSalvo et al., 2017). The thought of bringing users as close to the project development tends to bring them joy as they deem the technology is then developed based on all their requirements, concerns, ideas and suggestions. Participatory design occurs in the early stage of the software development cycle. The process of conducting participatory design is considered cheap as no coding is involved. Thus the overall project cost can be reduced by only paying programmers to develop only after the design of the technology is secure.

The aim of participatory design is evaluation. That said, the prototype or concept design was iteratively designed and evaluated to improve problems found in the original design. Therefore, the shared views and concerns raised during the participatory design sessions are essential, especially during design. Human-Computer Interaction (HCI) studies conducting participatory design has proven that by incorporating users as the "designers" during the design stage, the usability of the technology is often positive.

For example, a study by Razak et al. (2013) shows that including a number of older adults in a participatory design to develop a medicine reminder system brings positive outcomes during the field study. Fewer usability problems have been found in each stage of the design and development. Lee et al. (2017) conducted a participatory design to design a robotic technology with older adults with depressions. They conducted a few stages of participatory design involving all stakeholders, including many older adults, clinicians and caretakers. Rich data have been gathered to design the robotic technology, including the methodological aspect of conducting participatory design. Another participatory design by Ahmed et al. (2019) found that the method works perfectly in order for the researcher to develop a dashboard system for the cardiac patient. Similarly, this study conducted participatory design sessions with new numbers of older adults and clinicians.

\section{Number of Participants per Participatory Design}

There are mixed views in the literature about the optimal number of participants in a participatory design. In the Handbook of Participatory Design, the authors highlighted having several people join a participatory design to get a broader view or ideas of evaluating 
such a product (Simonsen \& Robertson, 2012). Fewer participants may influence the number of data gathered compared to having a large number of participants. However, no exact figure was given. A survey paper by Bossen et al. (2016) also did not highlight the optimum number of participants in the 17 participatory design studies they reviewed. The authors focused more on the result and the process to run the participatory design study.

In the early days, taken nearly a decade back, in Razak et al. (2013) study, as stated above, they recruited six older adults in six different sessions to achieve the paper's objective. That being said, they had one older adult per one participatory design session. However, just a year later, in a study by Wilkinson and De Angeli (2014), they recruited 25 participants (16 males, 9 females) in one session to investigate designing a walkingaid intelligent mobility device to assist in daily activities, for example, shopping in a shopping complex. The session includes discussing how shopping means for them and how technology can assist them in shopping effectively.

In Van Vensen et al. (2015), they recruited seven participants (no gender given) in one session to discuss a technology design concept for detecting and preventing frailty among older adults. However, in a study by Šabanović et al. (2015) to design socially assistive robots for older adults, they conducted two workshops to 1) learn what older adults think about technology and 2) design technology that suits them. In the first workshop, the authors had four participants ( 3 males, 1 female). In the second workshop, they had three participants ( 2 males, 1 female).

In recent studies of engaging older adults in participatory design by Ahmed et al. (2019), the authors recruited seven older adults in one participatory design session to design a dashboard system for the cardiac patient. In LaMonica et al. (2021) study, they had 21 older adults in four participatory design sessions. Unfortunately, no breakdown of the number of participants in each session was given.

To the best of our knowledge, after reviewing the literature, although all papers succeeded in using participatory design to achieve their objectives, no specific reason was given as to why the authors had this number of participants. The numbers varied from having one participant per participatory design study to 25 per session. There is also no definite number of participants for participatory design concerning the age group of the participants, especially with older adults. It shows that limited participatory design studies investigated the number of participants that suits a participatory design session. The mixed views on the optimal number of participants per participatory design prompt us to explore more in this area and how this affects the quality of the evaluation elicited. We also hoped to provide reflections on how best to conduct participatory design with older adults.

We are interested in designing and developing apps for older adults to address malnutrition, dehydration, and loneliness. Unfortunately, these health areas are too private and personal for a community of older adults to admit that they are malnourished or lonely. 
Thus, we are interested in investigating the use of two participatory designs to design two apps. The first participatory design is to be conducted individually between one older adult and the moderator, following what was practised by Razak et al. (2013) to address malnourishment. The second participatory design to address loneliness to be conducted in groups, following what was practised by Šabanović et al. (2015).

This paper is organised as a section of the methodology, followed by the results and discussions, and lastly, conclusion of the paper.

\section{METHODOLOGY}

\section{Design}

Two participatory design studies were conducted to achieve this. These two app suggestions derive from our previous study, which can be found in Sani et al. (2020). One participatory design (hereafter "PD1") with three older adults was to individually design a web app to self-monitor their fruit, vegetable and liquid intakes. The second participatory design (hereafter "PD2") was with a group of four older adults to design a smartphone app to address loneliness.

For both studies, a concept design storyboard of how the app might work was illustrated to the participant. That said, for PD1, a concept design to self-monitor fruit and vegetable intakes were provided and for PD2, a concept design to address loneliness was presented. The concept design was created following heuristics and guidelines for developing applications for older adults (e.g. Silva et al., 2015; Baharum et al., 2018; Sani \& Petrie, 2019). The concept designs were then evaluated for usability with experts prior to the study with the older adults. It makes sense to allow the experts to identify potential usability problems before evaluating the system to the older adults.

This study evaluated the concept design in three sessions to the participants in PD1 and one session for PD2. Both studies were moderated by the same moderator who has experience in research with older adults.

\section{Participants}

The inclusion criteria were to be 55 years or over, as this is still a choice of retirement age in Malaysia, and to live independently, either alone or with family members. That being said, we did not recruit older adults who have a carer or one who cannot manage to take care of themselves. Participants did not have to have any technology experiences. Participants were recruited from Pusat Aktiviti Warga Emas (PAWE), a social centre for older adults in Malaysia. In addition, participants were recruited from PAWE Labuan and PAWE Kota Kinabalu. All participants own and use smartphones. None of the participants was related to one another. Table 1 summarises the participants demographic. 
Table 1

Demographic of the participants

\begin{tabular}{lcc}
\hline Characteristic & PD1 & PD2 \\
\hline Gender & 2 women, 1 man & 2 women, 2 men \\
Age & $60-63$ years & $62-65$ years \\
Living Arrangement & All living with partner / family & All living with partner / family \\
Employment Status & All retired & All retired \\
\hline
\end{tabular}

\section{Procedure}

The procedure was the same for both studies. Malay and English languages were used to ease the participants. For PD1, the session started one by one with 10 minutes gap in between. Each session lasted approximately 45 mins. For PD2, one session was conducted with all four participants discussing together. The session was approximately 110 mins. During the studies, there were no other activities at PAWE.

The moderator started the study by introducing the objectives and procedures of the study. Participants were welcome to ask any questions prior to the study if they had any confusion. The moderator then displayed the concept design of the app. The participants were then asked to think aloud their thoughts and concerns about the design. The participants were also asked to give design suggestions and improvements if they deemed needed to motivate them to use the app in the future.

At the end of the study, the moderator highlighted all the key points reported by the participants. The participants were also debriefed. Finally, pack lunch meals and a monetary reward was given to each participant to thank them for their time and efforts.

\section{Materials and Equipment}

Two concept design storyboards were printed screen by screen on A4 papers. Sample of the concept design for PD1 and PD2 is as shown in Figures 1 and 2, respectively. In addition, each participant was given a copy of the concept design storyboard for both sessions to stimulate the discussion.

Participants were also provided with A4 papers for them to scribble their design suggestions and concerns. In addition, pens and markers were scattered around the table to reach them when needed easily.

The studies were audio-recorded using the Voice Recorder application on Samsung Note 9, running on Android 10.

\section{Data Analysis}

The audio recordings were transferred to iTunes for transcriptions and analysis of data. The transcriptions were translated to English by a translator. 

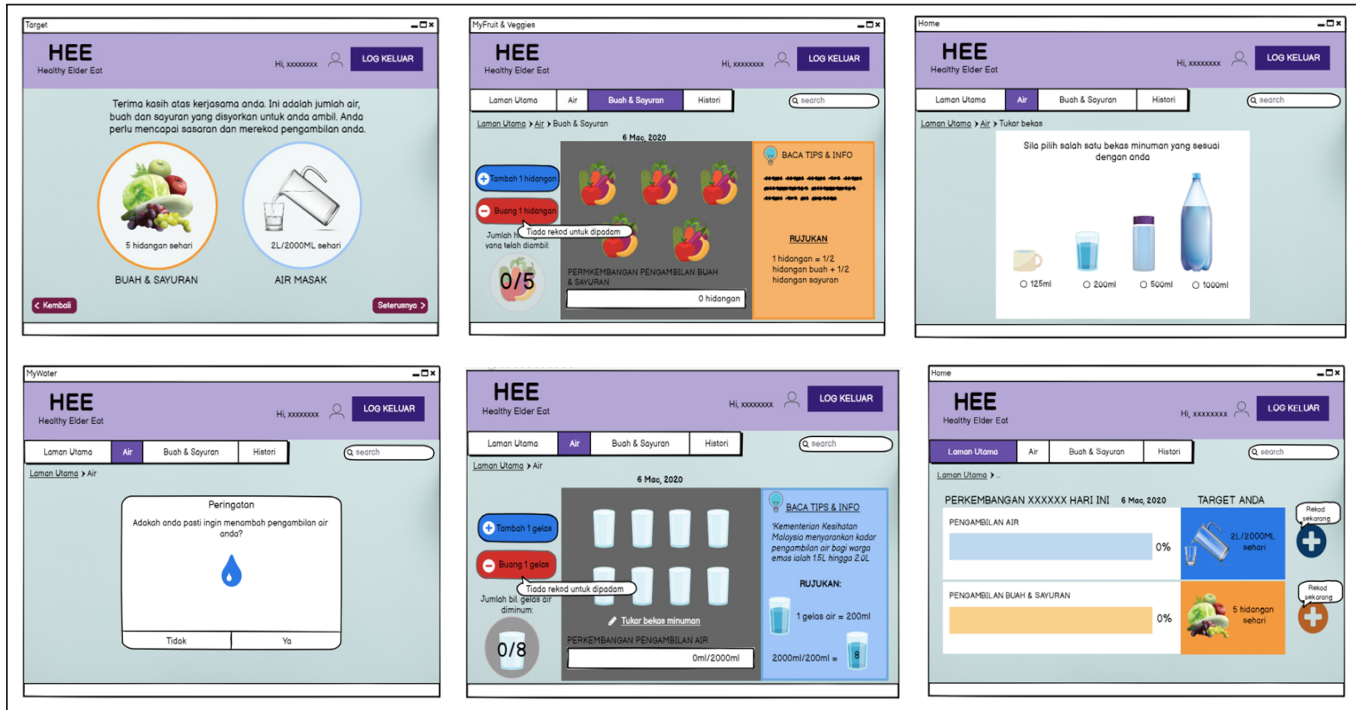

Figure 1. Sample of the concept design for PD1; an app to self-monitor fruit, vegetable and liquid intakes
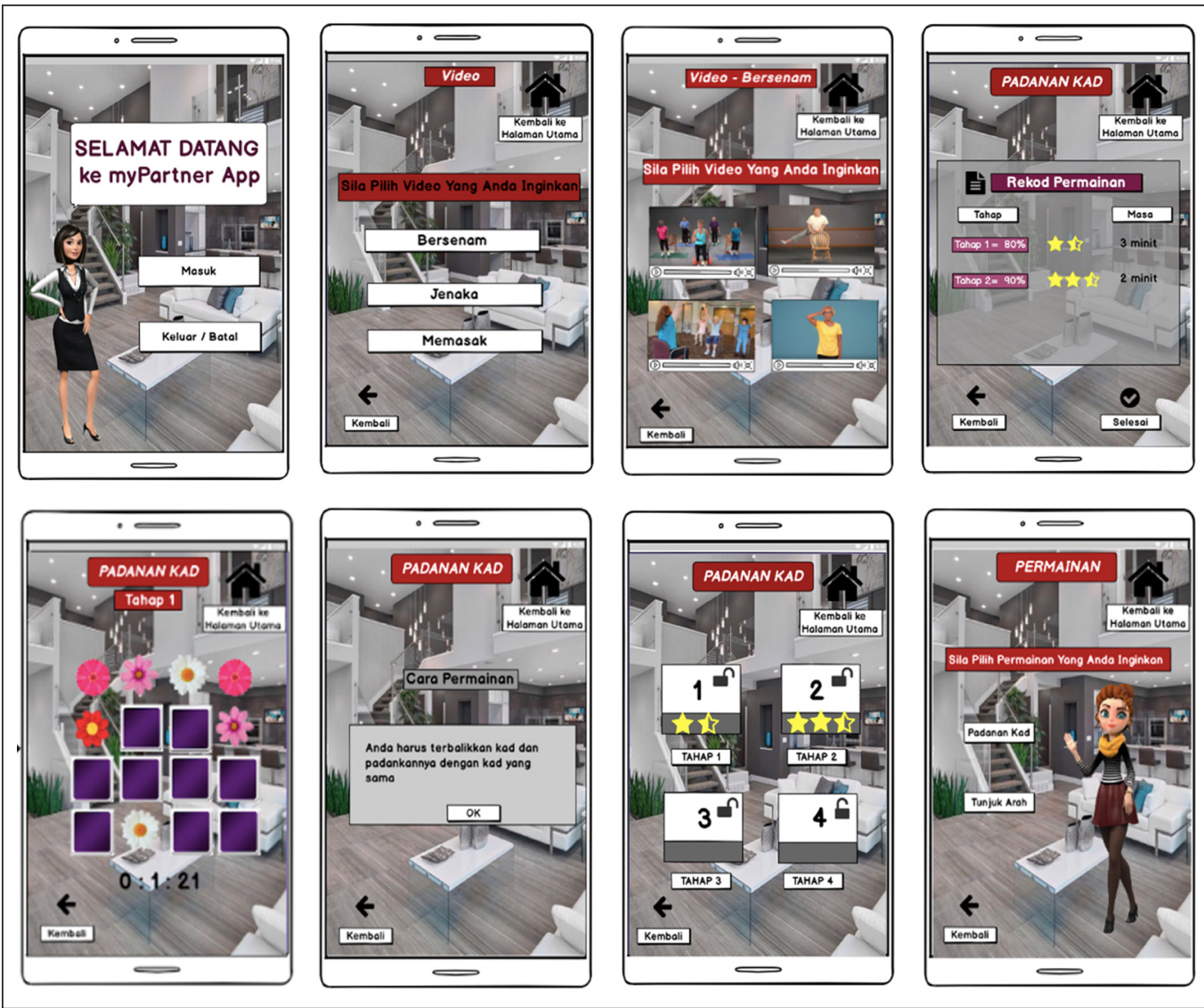

Figure 2. Sample of the concept design for PD2; an app to address loneliness 
Thematic analysis was conducted on the transcripts (Terry et al., 2017). It was to identify all suggestions, ideas, and concerns related to older adults in using the apps. First, an open coding technique was done until refined themes were found (Williams \& Moser, 2019). Then, to ensure the inter-coder reliability of the themes, a second coder went through all of the suggestions, ideas, and concerns and any disagreements were resolved.

\section{RESULT}

The studies provided insights that older adults can articulate their wants and needs in designing technology. For example, in both PD1 and PD2, we repeatedly found similar themes across both studies regarding having a technology that suits this particular group.

\section{Awareness of Personal Limitation}

Older adults have a certain ability to remember as their cognitive ability tends to decline as they get old. The older adults in this study were aware of their limitations in achieving a certain goal. It was repeatedly said in both PD1 and PD2. Some of the comments were:

- "I do not think I can remember all those steps" PD1.p1

- "The memory game requires a lot of attention I need time to learn that" PD2.p3

- "The exercise should be suitable for us we are sixty plus we cannot do like the younger people do" PD2.p1

- "That message was good it is needed so we know what we just did you know sometimes we tend to forget" PD1.p1

\section{Self-doubt and Concerns}

Literature has shown that older adults have positive and negative attitudes towards technology (e.g. Anderson \& Perrin, 2107; Elueze \& Quan-Haase, 2018). Similar to the findings in the literature, some older adults in this study also doubt certain features of the app and their capability to use it. Some of the comments were:

- "well I guess you know that we are not well adapt to using technology changes can be made but I think to get everyone to like using it is a different story" PD1.p3

- "you were born in a different era than us we grew up with no technology whatsoever I only started seeing this when I work I mean a few years working already then only we had computers the big ones so what I am trying to say is this changes it takes time maybe for me maybe not for me" PD2.p2

\section{Encouraging Interactions}

Literature has shown that older adults are prone to using such technologies if given sufficient and not burden instructions (Betts et al., 2019). The older adults in this study reported 
needing encouraging interactions that can assist them when required, especially if they feel lost upon using the technology. Some of the comments were:

- "I was a bit lost just now maybe you can have a little note to tell us where are we now" PD2.p2

- "so now what will happen how can I see what I just drank maybe you can direct me to that page or at least give me a message" PD1.p1

- "this is useful when you keep on giving us those messages I can see it helps us I like them" PD1.p2

\section{Learnability}

The older adults in this study prefer to use technology that does not require them to learn a lot. Instead, they suggest having features that are similar or familiar to them. It can be seen to reduce their mental workload to learn something new. Some of the comments were:

- "The overall flow of adding intakes should be made easy" PD1.p3

- "Why not you do something that is familiar to us but in a technology way I mean for example the " PD1.pl

- "The layout should be consistent and similar among all pages if a button is there at this page it should be there at the next page with the same size same location as an older person it will be easier for us to remember if there is not a lot of changes between the pages" PD2.p3

- "limit the amount of features to do per page please I cannot remember what to do if you give me all of those in one go" PD1.p2

\section{Privacy and Personal Matters}

We are aware that the topics between the two studies are different. We noticed that the older adults in PD1 are more open to discussing from their perspective. However, in PD2, the older adults often discuss by referring themselves to a friend or partner. Some of the comments were:

- "I want to use it cause I want to know my daily intakes I can also compare my whole week or months right" PD1.p3

- "I cannot lie I do not really drink water but I think this can help me to monitor my intake" PD1.p2

- "if you ask me know I can only say from the perspective of other older adults because I do not think that I am lonely" PD2.p3

- "Like my friend she lives alone she never says she is lonely but I think she is especially at night her children lives far away and she lost her husband I think this app can be useful for her she can spend time using it" PD2.p1 
- "for example my husband he is a lot older than me he likes to stay at home I sometimes go out with my girl friends we go mengaji like that so I think like my husband he can use this to fill in his free time rather than just watching tv right when I am not around" PD2.p4

- "maybe you can include a chatting section or video call features like whatsapp right I am not that lonely but I do think communication is important to reduce loneliness my sister living far in our village might need this app she can play the game or do some exercise to spend some time alone right" PD2.p3

\section{Older Adults as Designers}

Both PD1 and PD2 were discussing health app designs that might help older adults in their daily life. We acknowledge that both topics were significant health issues among older adults (BDA, 2021).

In PD1, the moderator had to provoke the session more to encourage the design suggestions, which was not a surprise as this was a one-to-one discussion. In PD2, the participants built up the discussion and suggestions but were closely monitored by the moderator to avoid the discussion going out of topic or one participant conquering the discussion.

In PD1, we noticed that involving the moderator directly with the participants, allows them to articulate their design concerns and needs eagerly. Unfortunately, compared to PD2, some design suggestions or concerns were supported or rejected by the participants. However, the moderator resolved it by reminding us that all suggestions or concerns were welcome in the discussion.

In regards to time, four sessions of PD1 were approximately $60 \mathrm{mins}$ per participant. For PD2, the session lasted for $110 \mathrm{mins}$. Therefore, it can be said as $27.5 \mathrm{mins}$ per participant to articulate their thoughts and concerns on the discussion. We did not count the number of contributions per participant as it was not in the interest of this paper.

\section{DISCUSSIONS AND CONCLUSION}

This paper investigated the use of two methods on participatory design for working with older adults to design and evaluate technologies to support independent living. That being said, our study focuses on the mutual learning concept between researchers and the older adults as users.

For PD1, we asked about an app to self-monitor fruits, vegetables and liquid intake. For PD2, we asked about an app to address loneliness. We found that both PDs brings rich data to our research. However, in PD1, the moderator may need to put effort to encourage the 
discussion. It is not a big issue as participants need time to warm up prior to the discussion. In PD2, although the participants did not know each other, they managed to sit down and discuss the topic together. No major issue was raised during PD2. All participants were given time to speak, raised concerns and gave suggestions to design the app.

We acknowledged that the topic of discussions might be too personal or private. However, the participants in both PDs did not claim that they were lonely or malnourished. Their health condition is also not in the interest of the PDs. In PD2, the participants refer to loneliness by referring to themselves from a friend or partner's point of view. In PD1, by just having the moderator and the participant in each session, the participants seem to be more open and gave concerns from their point of view. It is, however, not the interest of this paper to compare the amount of contribution per participant in regards to the same topic, individually or in a group.

We understand the fact that older adults are less experienced in using technology as compared to younger adults. Thus, we recommend that the moderator be well adept and experienced in research with older adults. Although there was no issue in the study, moderators may need to explain more, especially in the designs and interfaces that are not familiar with the older adults. In particular, we found that the older adults in PD2 did not like the interactions with humour videos, which further investigation can be done. We also acknowledge that the participants in this study have a socio-economic of the somewhere middle class. Thus, the results might differ if we had a range of participants.

We are also aware of the limitations relating to the methodology, and the conclusion drawn from this paper should be taken with these in mind. Finally, we acknowledge that although this current study produced enough data for us to develop the apps, this study has a very minimal number of participants that are unable to represent the whole targeted population.

Overall, either alone or in a group, both methods show that older adults can articulate their wants and needs in designing technology. Therefore, we recommend using this method in regard to designing technologies for older adults. Future work can perhaps investigate preference PD based on the topic to be discussed. It is unclear from this study if the topics in both PDs interfere with the contribution per discussion.

\section{ACKNOWLEDGEMENTS}

University Malaysia Sabah funds this research under the grant SPLB0180-2018 led by the first author. We want to thank all participants in this study for their time and effort. We would also like to thank the first author's Final Year students for assisting in designing and developing the apps. 


\section{REFERENCES}

Ahmed, R., Toscos, T., Ghahari, R. R., Holden, R. J., Martin, E., Wagner, S., \& Mirro, M. (2019). Visualization of cardiac implantable electronic device data for older adults using participatory design. Applied Clinical Informatics, 10(4), 707-718. https://doi.org/10.1055/s-0039-1695794

Anderson, M., \& Perrin, A. (2017). Technology use among seniors. Pew Research Center for Internet \& Technology.

Baharum, A., Ismail, R., Saad, N., Daruis, D. D. I., Noh, N. A. M., \& Noor, N. A. M. (2018). Development of elderly reminder mobile application using mental model. In Proceedings of the 2018 International Conference on Artificial Intelligence and Virtual Reality (pp. 131-136). ACM Publishing. https://doi. org/10.1145/3293663.3293665

Betts, L. R., Hill, R., \& Gardner, S. E. (2019). “There's not enough knowledge out there”: Examining older adults' perceptions of digital technology use and digital inclusion classes. Journal of Applied Gerontology, 38(8), 1147-1166. https://doi.org/10.1177/0733464817737621

Bossen, C., Dindler, C., \& Iversen, O. S. (2016). Evaluation in participatory design: A literature survey. In Proceedings of the 14th Participatory Design Conference: Full papers-Volume 1 (pp. 151-160). ACM Publishing. https://doi.org/10.1145/2940299.2940303

BDA. (2021). Loneliness and malnutrition. The Association of UK Dietitians

DOSM. (2020). Current population estimates, Malaysia, 2018-2019. Department of Statistics Malaysia.

DiSalvo, B., Yip, J., Bonsignore, E., \& DiSalvo, C. (Eds.). (2017). Participatory design for learning: Perspectives from practice and research. Taylor \& Francis.

Elueze, I., \& Quan-Haase, A. (2018). Privacy attitudes and concerns in the digital lives of older adults: Westin's privacy attitude typology revisited. American Behavioral Scientist, 62(10), 1372-1391. https:// doi.org/10.1177/0002764218787026

Hakobyan, L., Lumsden, J., Shaw, R., \& O’Sullivan, D. (2016). A longitudinal evaluation of the acceptability and impact of a diet diary app for older adults with age-related macular degeneration. In Proceedings of the 18th International Conference on Human-Computer Interaction with Mobile Devices and Services (pp. 124-134). ACM Publishing. https://doi.org/10.1145/2935334.2935356

LaMonica, H. M., Davenport, T. A., Roberts, A. E., \& Hickie, I. B. (2021). Understanding technology preferences and requirements for health information technologies designed to improve and maintain the mental health and well-being of older adults: Participatory design study. JMIR Aging, 4(1), Article e21461. https://doi.org/10.2196/21461

Lee, H. R., Šabanović, S., Chang, W. L., Nagata, S., Piatt, J., Bennett, C., \& Hakken, D. (2017). Steps toward participatory design of social robots: Mutual learning with older adults with depression. In Proceedings of the 2017 ACM/IEEE international conference on human-robot interaction (pp. 244-253). ACM Publishing. https://doi.org/10.1145/2909824.3020237

Mehra, S., Visser, B., Dadema, T., Van Den Helder, J., Engelbert, R. H., Weijs, P. J., \& Kröse, B. J. (2018). Translating behavior change principles into a blended exercise intervention for older adults: Design study. JMIR Research Protocols, 7(5), Article e117. https://doi.org/10.2196/resprot.9244 
Razak, F. H. A., Razak, N. A., Adnan, W. A. W., \& Ahmad, N. A. (2013). How simple is simple: Our experience with older adult users. In Proceedings of the 11th Asia Pacific Conference on Computer Human Interaction (pp. 379-387). ACM Publishing. https://doi.org/10.1145/2525194.2525307

Šabanović, S., Chang, W. L., Bennett, C. C., Piatt, J. A., \& Hakken, D. (2015). A robot of my own: Participatory design of socially assistive robots for independently living older adults diagnosed with depression. In International conference on human aspects of it for the aged population(pp. 104-114). Springer. https:// doi.org/10.1007/978-3-319-20892-3_11

Sani, Z. H. A., \& Petrie, H. (2017). Evaluation of an app to support healthy living by older adults. Electronic Visualisation and the Arts (EVA 2017), 1-14. https://doi.org/10.14236/ewic/HCI2017.42

Sani, Z. H. A., \& Petrie, H. (2019). Older adults' number entry using touchscreen and keyboard-mouse computers. In International Visual Informatics Conference (pp. 353-367). Springer. https://doi. org/10.1007/978-3-030-34032-2_32

Sani, Z. H. A., Nizam, D. N. M., Baharum, A., \& Tanalol, S. H. (2020). Older adults' needs and worries about healthy living and mobile technology: A focus group study. International Journal of Advanced Science and Technology, 29(9s), 1127-1136.

Silva, P. A., Holden, K., \& Jordan, P. (2015). Towards a list of heuristics to evaluate smartphone apps targeted at older adults: a study with apps that aim at promoting health and well-being. In 2015 48th Hawaii International Conference on System Sciences (pp. 3237-3246). IEEE Publishing. https://doi.org/10.1109/ HICSS.2015.390

Simonsen, J., \& Robertson, T. (Eds.). (2012). Routledge international handbook of participatory design. Routledge.

Terry, G., Hayfield, N., Clarke, V., \& Braun, V. (2017). Thematic analysis. In C. Willig \& W. Stainton-Rogers (Eds.), The Sage handbook of qualitative research in psychology (pp. 17-37). Sage Publication Ltd.

Tulu, B., Strong, D., Wang, L., He, Q., Agu, E., Pedersen, P., \& Djamasbi, S. (2016). Design implications of user experience studies: the case of a diabetes wellness app. In 2016 49th Hawaii International Conference on System Sciences (HICSS) (pp. 3473-3482). IEEE Publishing. https://doi.org/10.1109/HICSS.2016.434

Van Velsen, L., Illario, M., Jansen-Kosterink, S., Crola, C., Di Somma, C., Colao, A., \& Vollenbroek-Hutten, M. (2015). A community-based, technology-supported health service for detecting and preventing frailty among older adults: a participatory design development process. Journal of Aging Research, 2015, Article 216084. https://doi.org/10.1155/2015/216084.

Wilkinson, C. R., \& De Angeli, A. (2014). Applying user centred and participatory design approaches to commercial product development. Design Studies, 35(6), 614-631. https://doi.org/10.1016/j. destud.2014.06.001

Williams, M., \& Moser, T. (2019). The art of coding and thematic exploration in qualitative research. International Management Review, 15(1), 45-55.

WHO. (2021). Ageing and health. World Health Organization. 
